On re-audit $32.5 \%$ more of discharge summaries were completed within 7 days of discharge. This enables GPs to carry out requests made e.g. repeat prescriptions, thus ensuring a safer discharge process.

Lessons learnt The whole departmental staff wasn't present at the induction session; those who weren't tended to make more omissions from their summaries, and were less efficient. We shall therefore run a session at the departmental teaching, as well as at induction, as part of the rolling audit.

Message for others We audited elements of the discharged summaries we felt necessary for a smooth and safe discharge from hospital to community care. By instructing new doctors on how to use the local computer program, we improved on the efficiency and degree of completion. A rolling audit has been started and further interventions will be made.

\section{G564(P) PRE-OPERATIVE SCREENING FOR SICKLE CELL DISEASE: IMPROVING PARENT AND CARER EXPERIENCES}

S Iqbal, E Wedge, M McPhee, E Chua. Paediatrics, Imperial College Healthcare NHS Trust, London, UK

\subsection{6/archdischild-2015-308599.513}

Context As part of hospital policy, all children deemed high risk for the carriage of sickle cell disease presenting to the paediatric pre-operative assessment clinic at are to be tested for the condition. This involves well children undergoing a blood test.

Problem It was found that considerable parental/carer anxiety was produced by this testing and much time was spent in a busy clinic counselling parents about the condition and the need for testing. Through the production of an information leaflet explaining sickle cell disease and the reason behind testing children prior to an operation, it was hoped parents/carers would feel more informed and thus more willing for their children to testing.

Assessment of problem and analysis of its causes Prior to implementation of the leaflet, of 21 parents/carers only two thirds (14) felt they fully understood the reason for testing $(5 / 5$ on qualitative scale) and only two thirds (14) felt fully happy for their child to have the blood test $(5 / 5) .71 \%$ of parents/carers (15) thought an information leaflet would be useful.

Intervention An information leafter was written by the authors, with expert review from a consultant paediatric haematologist. This passed the local procedure for patient information and was introduced to the clinic. Nursing staff handed out the leaflet to parents of children from at-risk ethnicities on arrival, giving parents time to read it before their consultation with the doctor. Study design Over four weeks all parents/carers of children requiring sickle cell screening presenting to the clinic completed a qualitative questionnaire about their attitude to screening. An information leaflet was subsequently designed aimed at improving parent/carer's knowledge about sickle cell disease prior to consenting for the test. Following implementation of the leaflet a repeat qualitative questionnaire was carried out to assess the leaflet's impact.

Strategy for change Collaboration and education with nursing staff played a vital role in integrating the leaflet into the standard practice of the clinic.

Measurement of improvement A repeat questionnaire was carried out after introduction of the leaflet. Unfortunately there were relatively few children requiring screening during this month (total 9). Of those screened, $100 \%$ of parents felt that the reason for testing was completely clear $(5 / 5$ on quantitive scale). $89 \%$ were fully willing for their child to have the blood test. $89 \%$ reported that they found the leaflet useful.

Effects of changes Subjectively, we have found that the consultations with parents are quicker and easier due to reduced parental anxiety.

Lessons learnt Patient informaiton leaflets are a useful method of improving communication with families and improving families' experience of the service in pre-operative assessment.

Message for others This was a small project but was successful and we hope that our experience will encourage others to develop patient information when screening policies are in place as this is an area where medical concerns may not line up with parents' concerns.

\section{G565(P) MULTI-PROFESSIONAL WORKFORCE TASK MAPPING IN GENERAL PAEDIATRICS}

K Orlowski, D Jyothish, S Agarwal, E Archary. General Paediatrics, Birmingham Children's Hospital NHS Foundation Trust, Birmingham, UK

\subsection{6/archdischild-2015-308599.514}

Context Task mapping exercises have a valuable part in shaping future workforces. Our project examined the tasks undertaken by a multi-professional, non-consultant workforce in a large, busy general paediatric department, which provides secondary level paediatric care and also assists in the care of tertiary subspecialty patients, providing a total of 12000 inpatient episodes of care per year.

Problem Uncertainties around numbers of doctors in training allocated to the department in the future and the anticipated contraction of the junior medical workforce had led to new models of care provision. Introduction of international fellows and non-medical practitioners such as advanced nurse practitioners and physician associates into the team are seen as a way of developing a resilient and future-proof workforce.

Assessment of problem and analysis of its causes There was a perception of mismatched distribution of competencies within our team, with an assumed deficiency in middle grade level skills. Therefore, we embarked on a task mapping project to examine the spectrum of everyday tasks undertaken by the team in order to ensure that the non-consultant workforce had the right competencies to deliver safe and timely patient care.

Intervention A series of initial stakeholder meetings were held, and a task mapping sheet developed which coded all clinical and non clinical tasks that could be anticipated to be performed over the working day. Each team member was required to enter a code at 15 min intervals, as well as a code for any pending tasks, daily for 3 weeks. A pilot was run with senior trainees in the department following which amendments were made. Champions for the project were identified, and educational sessions with power point presentations were given prior to commencing the project.

Study design The study used a Plan-Do-Study-Act model as a framework. The data collection sheet was partially anonymised, requiring the individual to complete only their clinical grade and location of work.

Strategy for change Data analysed shows the amount of time each team-member group spent on each coded task. There is a reliance on middle grade staff for practical procedures $-66 \%$ of lumbar punctures are first attempted by registrars, and $40 \%$ of intravenous access. Attendance rates at educational sessions are highest by FY trainees, with lowest rates by registrars. There needs to be a cultural change, with reallocation of tasks from 